\title{
Methylation of green tea polyphenols affects their binding to and inhibitory poses of the proteasome $\beta 5$ subunit
}

\author{
KENYON G. DANIEL ${ }^{1}$, KRISTIN R. LANDIS-PIWOWAR ${ }^{1}$, DI CHEN ${ }^{1}$, \\ SHENG BIAO WAN ${ }^{2}$, TAK-HANG CHAN ${ }^{2}$ and Q. PING DOU ${ }^{1}$
}

\begin{abstract}
${ }^{1}$ The Prevention Program, Barbara Ann Karmanos Cancer Institute, and Department of Pathology, School of Medicine, Wayne State University, 4100 John R Road, Detroit, MI, USA; ${ }^{2}$ Department of Applied Biology and Chemical Technology and the Open Laboratory for Chirotechnology and Central Laboratory, Institute of Molecular Technology for Drug Discovery and Synthesis, the Hong Kong Polytechnic University, Hong Kong SAR, China
\end{abstract}

Received March 20, 2006; Accepted April 24, 2006

\begin{abstract}
Previously, we showed that ester carbon-containing tea polyphenols, including (-)-epigallocatechin gallate [(-)EGCG] and (-)-epicatechin-3-gallate [(-)-ECG], potently inhibit proteasomal chymotrypsin-like activity. In addition, our in silico docking study suggested that a particular pose of (-)EGCG could lead to potential covalent modification of the Nterminal threonine (Thr 1) of the proteasome 35 subunit in the chymotrypsin-like active site. It has been suggested that some major biotransformation reactions, such as methylation, could result in reduced biological activity of (-)-EGCG in vivo. We hypothesize that methylation reduces binding of (-)-EGCG to the $B 5$ subunit of the proteasome and, therefore, decreases its proteasomal chymotrypsin-like-inhibitory potency. Here, we report that, while methylation has no effect on nucleophilic susceptibility of (-)-EGCG and (-)-ECG, it may disrupt the ability of these polyphenols to interact with Thr 1 of the proteasome $B 5$ subunit. In silico docking shows that methylation results in the tea polyphenols' ester carbon being moved away or blocked entirely from Thr 1 . Additionally, methylation impairs the ability of (-)-EGCG and (-)-ECG to dock in a consistent low energy pose. These observations, no change in nucleophilic susceptibility, moving or blocking the ester carbon from Thr 1, and lack of a consistent docking pose, suggest that methylation disrupts the ability of (-)-EGCG and (-)-ECG to bind to the proteasome 35 subunit, which may then diminish their proteasomal chymotrypsin-inhibitory and, therefore, other biological activities.
\end{abstract}

Correspondence to: Dr Q. Ping Dou, Department of Pathology, School of Medicine, Wayne State University, 4100 John R Road, Detroit, MI 48201, USA

E-mail: doup@karmanos.org

Key words: tea polyphenols; (-)-epigallocatechin gallate, (-)-epicatechin-3-gallate, methylation, proteasome inhibitors, cancer, docking, computer modeling

\section{Introduction}

Green tea polyphenols, in particular (-)-EGCG, have been suggested to have cancer-preventative and anticancer effects (1-5). Although the involved molecular targets have not been clearly defined, one candidate is the proteasome (6-9). We have reported that ester carbon-containing tea polyphenols, especially (-)-ECG and (-)-EGCG, are potent inhibitors of the proteasome and the ester carbon appears to be necessary to facilitate this inhibition (9). Furthermore, by using both natural and synthetic ester bond-containing tea polyphenols, we found that the ester carbon in these compounds is highly susceptible to nucleophilic attack and that (-)-ECG and (-)EGCG may inhibit the chymotrypsin-like activity of the proteasome possibly through covalent modification of the N-terminal threonine (Thr 1) $(9,10)$. This could be accomplished via nucleophilic attack of a pair of electrons from the hydroxyl group of Thr 1 to the ester carbon of (-)-ECG or (-)EGCG (10). In silico modeling experiments demonstrated a strong site of nucleophilic attack at the ester carbon and a conserved pose in which (-)-ECG and (-)-EGCG filled the active site and placed the ester carbon in close proximity to Thr 1 (10). A series of (-)-EGCG analogs were also docked, further validating the model (10).

Although clinical, animal, and epidemiological studies have shown the anticancer benefits of (-)-EGCG and tea polyphenols $(7,11,12)$, the biological activities of these tea polyphenols could be reduced in vivo by several biotransformation reactions, including methylation $(13,14)$. However, the molecular consequences for tea polyphenol inactivation by methylation reactions are unknown. We most recently discovered that methylation could decrease the proteasomeinhibitory effects of tea polyphenols in vitro and in cultured tumor cells (unpublished data).

We hypothesize that methylated (-)-ECG or (-)-EGCG have decreased binding affinity to the proteasome $\beta 5$ subunit, which could be responsible for their decreased potencies to inhibit proteasomal chymotrypsin-like activity (unpublished data). To test this hypothesis, we examined the effects of methylation on nucleophilic susceptibility, binding pose, and 
energy of green tea polyphenols (-)-ECG and (-)-EGCG. This was compared to the $\mathrm{IC}_{50}$ values of synthesized methylated analogs against a purified proteasome (unpublished data). We report here that, while methylation had no effect on the nucleophilic susceptibility of the (-)-ECG and (-)-EGCG ester carbon, it seems to have significant effects on their binding to the proteasomal 35 subunit. First, the poses adopted by the methylated polyphenols move the ester carbon a considerable distance away from Thr 1 or block the ester carbon from binding to Thr 1. Second, increased levels of methylation correlate with decreased ability of (-)-ECG and (-)-EGCG to bind to the active site in a predictable manner. Consistently, biological testing showed that increased methylation resulted in a dramatic increase in the $\mathrm{IC}_{50}$ values for proteasome inhibition (unpublished data). This information collectively, suggests that even single methylation events, if in the right position, such as paramethylation on the D-ring, can dramatically interfere with the ability of (-)-ECG or (-)-EGCG to bind to the proteasome. Therefore, loss of proteasome-binding by methylated analogs of (-)-ECG or (-)-EGCG, but not changes in nucleophilic susceptibility, may account for the reduced proteasomeinhibitory activity.

\section{Materials and methods}

Molecule construction and nucleophilic susceptibility analysis. Molecules were constructed using the CAChe Workstation (Fujitsu, inc.). Susceptibility to nucleophilic attack was determined by using the programs in CAChe with the PM5 geometry and PM5 wave function in water as the parameters. Molecules were subjected to geometry optimization using the PM5 wave function in water and saved in PDB format using the conversion filters in CAChe. The output PDB files were imported into AutoDock for ligand preparation as described below.

In silico binding analysis to the proteasome 35 subunit. The crystal structure of the eukaryotic yeast 20S proteasome was obtained from the protein database (15) (ref. no 1JD2), and used for all of the docking studies presented here. The yeast $20 \mathrm{~S}$ proteasome is structurally very similar to the mammalian $20 \mathrm{~S}$ proteasome and the chymotrypsin active site between the two species is highly conserved $(16,17)$. The AutoDock 3.0 suite of programs, which was used for the docking calculations, employs an automated docking approach, allowing ligand flexibility as described to a full extent elsewhere (18). AutoDock has been compared to various docking programs in several studies and has been found to be able to locate docking modes that are consistent with X-ray crystal structures $(18,19)$. Default parameters (including a distance-dependent dielectric 'constant') were used as described in the AutoDock manual except for those changes mentioned below.

The dockings were run on an i386 architecture computer running Redhat ${ }^{\mathrm{TM}}$ Linux 9.0. The crystal structure of the $20 \mathrm{~S}$ proteasome and the ligands were prepared for docking by following the default protocols, except where noted. The energy-scoring grid was prepared as a 40x40x40 $\AA$ box centered on the 35 catalytic Thr 1 , and the ligand was limited to this search space during docking. Atomic solvation parameters were assigned to the proteasome using default parameters. The default parameters for the lamarckian genetic algorithm (20) were used as the search protocol, except for the number of genetic algorithm runs, which was set to 100 , and the maximum number of energy evaluations, which was set to 5 million (the population size was retained at 50). AutoDock relies upon an empirical scoring function that provides approximate binding free energies. AutoDock reports a docked energy that we have referred to as a 'docked free energy'; since it includes a solvation free energy term. The docked energy also includes the ligand internal energy, or the intramolecular interaction energy of the ligand. AutoDock also reports a binding free energy that excludes the ligand internal energy but includes a torsional free term for the ligand based on the number of rotatable bonds.

In the present study, we chose to use the docked free energies since the number of rotatable bonds in our inhibitors is relatively constant and because we believed that the internal energy of the ligand should not be neglected for our compounds. Its neglect is tantamount to assuming that the intramolecular interaction energy of the ligand is the same in the complex as in the solution. It is worth noting that the docked free energies (or binding free energies) that one obtains may vary depending upon the precise force field parameters in use (e.g., charges, electrostatic treatment, etc.). For the GA algorithm, the default parameters were kept for mutation, crossover and elitism. The pseudo-Solis and Wets local search method was included using default parameters.

The output from AutoDock was rendered with PyMOL (21). The pose chosen for analysis was the most represented pose, typically the cluster with the greatest number of members. Due to the similarity of the molecules and docking scores we also examined the predictability of the poses. For this study, predictability of a pose is based on: (a) the total number of different poses that the molecule adopts during a dock out of 100 possibilities, (b) the number of times the molecule found an inhibitory pose out of 100 and (c) how many different poses are inhibitory. When ' $a$ ' is low, ' $b$ ' is high, and 'c' is low. This suggests that AutoDock could find a consistent binding pose and, thus, a predictable dock. As 'a' increases, 'b' decreases, and 'c' increases, which suggests that AutoDock was unable to find a predictable pose within the given parameters. An inhibitory pose is defined as one that places the ester carbon within $4.2 \AA$ of the oxygen of Thr 1; the slightly extended distance allows for protein flexibility.

\section{Results}

Methylation reduces the IC50 values of (-)-ECG and (-)-EGCG against a purified 20S proteasome. The structures of (-)-ECG and (-)-EGCG and their methylated metabolites are presented (Fig. 1). Unmethylated (-)-ECG and (-)-EGCG have $\mathrm{IC}_{50}$ values of 0.71 and $0.21 \mu \mathrm{M}$ respectively (unpublished data). A single methylation event could raise the $\mathrm{IC}_{50}$ by up to 35 -fold (Fig. 1). These $\mathrm{IC}_{50}$ values worsen as the number of methylation events increase, which will be reported in full elsewhere (unpublished data).

Methylation has no effect on the susceptibility of (-)-ECG and (-)-EGCG to nucleophilic attack. The ester carbon of green tea polyphenols has been shown to bestow proteasome- 
A<smiles>O=C(Oc1ccc(O)cc1)c1cc(O)c(O)cc1C(O)c1ccc(O)c(O)c1</smiles>

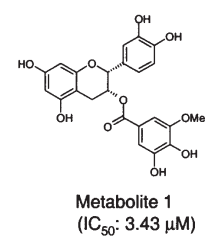

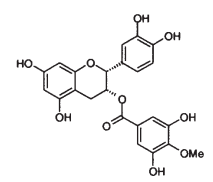

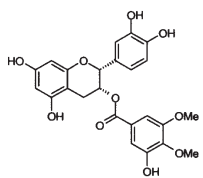

\section{Metabolite 2} $\left(\mathrm{IC}_{50}: 19.12 \mu \mathrm{M}\right)$ $\left(\mathrm{IC}_{50}: 48.25 \mu \mathrm{M}\right)$

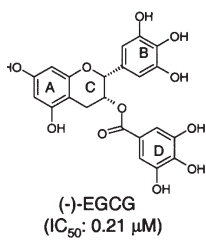

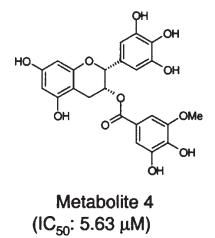

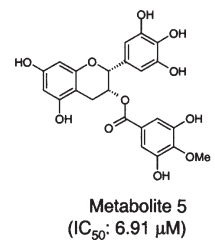

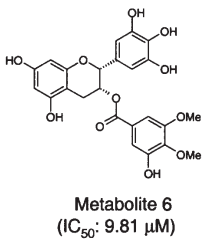

B

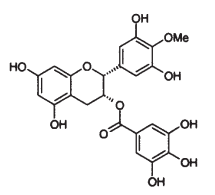<smiles>CCOc1c(O)cc(C(=O)OC2Cc3c(O)cc(O)cc3OC2c2cc(O)c(O)c(O)c2)cc1O</smiles><smiles>COc1cc(C(=O)OCC2Oc3cc(O)cc(O)c3Oc3cc(C)c(O)cc32)cc(O)c1O</smiles>

Metabolite 9 (IC $50: 43.03 \mu \mathrm{M}$ )

Figure 1. Structures of tea polyphenols, methylated metabolites, and their $\mathrm{IC}_{50}$ values to a purified $20 \mathrm{~S}$ proteasome.

inhibitory properties to these compounds (9). Since methylation significantly worsened the $\mathrm{IC}_{50}$ of the polyphenols (unpublished data), we first examined whether methylation has any effect on the potential for these compounds to undergo nucleophilic attack by Thr 1 (Fig. 2). In each case, we found that methylation had no apparent effect on the nucleophilic susceptibility of the ester carbon (Fig. 2). This suggested that the loss of proteasome-inhibitory activity in methylated polyphenols (unpublished data) might not be due to changes in their nucleophilic susceptibility.

Paramethylation or dimethylation on the D-ring of (-)-ECG results in a pose that moves the ester carbon distal from Thr 1 . Three methylated (-)-ECG analogs, named Metabolites 1-3, respectively (Figs. 1A and 3) were docked and cluster analysis was performed. Metabolites 1 and 2 each possess a single methyl group on the D-ring: Metabolite 1 is metamethylated, whereas Metabolite 2 is paramethylated (Fig. 1A). Metabolite 3 is dimethylated in both the meta and para positions on the Dring. Unmethylated (-)-ECG docks with 53 poses, placing the ester carbon in a good position to undergo nucleophilic attack with very high predictability. Analysis of the dock indicated that 26 different poses were found in total and 13 were potentially inhibitory (10). However, different types of methyl-

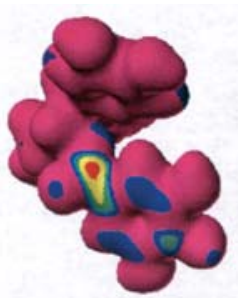

$(-)$-ECG

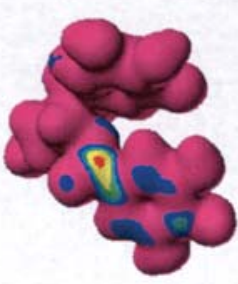

(-)-EGCG

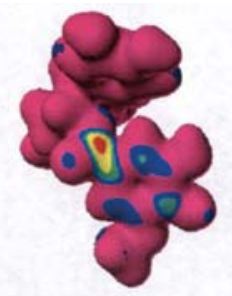

Metabolite 1

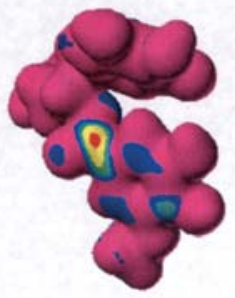

Metabolite 4

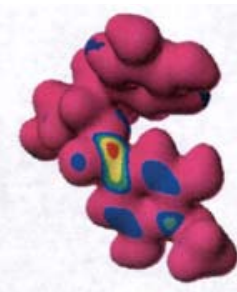

Metabolite 2

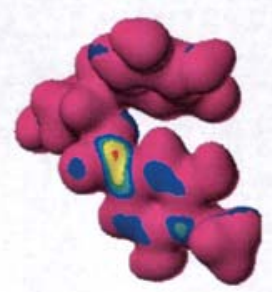

Metabolite 5

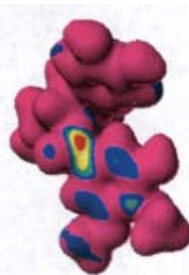

Metabolite 3

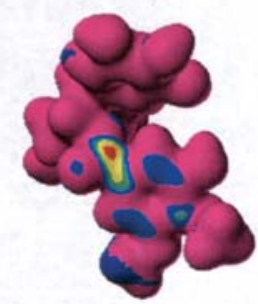

Metabolite 6

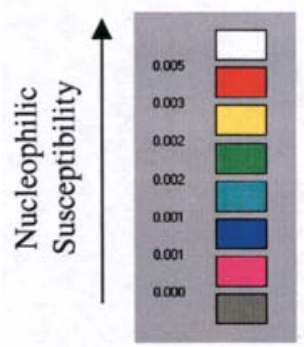

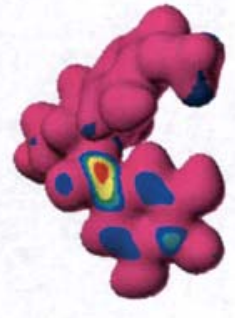

Metabolite 7

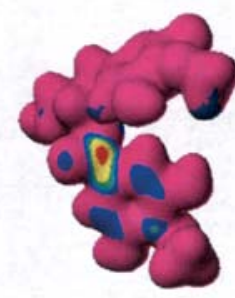

Metabolite 8

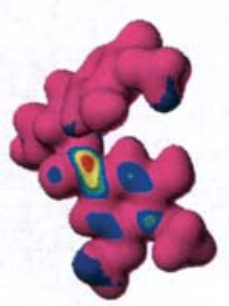

Metabolite 9

Figure 2. Nucleophilic susceptibility analysis of (-)-ECG, (-)-EGCG, and methylated metabolites. Nucleophilic susceptibility analysis was performed with CAChe using the PM5 geometry and wave function in water. The methylation events did not change the susceptibility of the ester carbon to undergo nucleophilic attack. 
Table I. Comparison of clusters for methylated (-)-ECG and (-)-EGCG analogs.

\begin{tabular}{lcccc}
\hline $\begin{array}{l}\text { Natural and } \\
\text { methylated } \\
\text { polyphenols }\end{array}$ & $\begin{array}{c}\text { Total number of } \\
\text { different poses (a) }\end{array}$ & $\begin{array}{c}\text { Total number of } \\
\text { inhibitory poses (b) }\end{array}$ & $\begin{array}{c}\text { Number of different } \\
\text { inhibitory poses (c) }\end{array}$ & $\begin{array}{c}\text { Predictability } \\
\text { out of 100 }\end{array}$ \\
\hline $\begin{array}{l}\text { (-)-ECG } \\
\text { Metabolite 1 }\end{array}$ & 26 & 53 & 13 & Very High \\
Metabolite 2 & $31 \uparrow$ & 52 & $15 \uparrow$ & High \\
Metabolite 3 & $41 \uparrow$ & $40 \uparrow$ & $15 \uparrow$ & Low \\
(-)-EGCG & $47 \uparrow$ & $48 \uparrow$ & 4 & Very Low \\
Metabolite 4 & 8 & 58 & $16 \uparrow$ & Very High \\
Metabolite 5 & $32 \uparrow$ & $56 \uparrow$ & $8 \uparrow$ & High \\
Metabolite 6 & $28 \uparrow$ & $46 \uparrow$ & $8 \uparrow$ & High \\
Metabolite 7 & $35 \uparrow$ & $64 \uparrow$ & $10 \uparrow$ & High \\
Metabolite 8 & $24 \uparrow$ & $69 \uparrow$ & $16 \uparrow$ & High \\
Metabolite 9 & $34 \uparrow$ & $55 \uparrow$ & High \\
\hline
\end{tabular}

Arrows indicate change in value compared to the parental compound. (a) Total number of distinct solutions $>1 \AA$ in RMS difference; the lower this number, the more predictable the docking. (b) The number of times the molecule docked with the ester carbon $<4.2 \AA$ away from the oxygen of Thr 1; the higher this number, the better chance the molecule can bind in an inhibitory manner. (c) As this number increases, it suggests that AutoDock was less successful at reproducing any given inhibitory pose. Predictability: a qualitative statement based on (a), (b), and (c); also considered, but not listed, is how many poses are low number and single member.

ation result in dramatic shifts of the pose (Fig. 3A-C). Furthermore, addition of methyl groups increase the total number of possible poses, thus decreasing the probability of the molecule adopting any given pose (Table I).

The docking pose most adopted by Metabolite 1 places the ester carbon at an appropriate distance for nucleophilic attack, $3.49 \AA$ (Fig. 3A). Metamethylated Metabolite 1 docks with 52 poses placing the ester carbon in good position to undergo nucleophilic attack (31 different poses in total and 15 of which were potentially inhibitory), suggesting a high degree of predictable binding (Table I). This, however, was worse than (-)-ECG. (-)-ECG possesses a high degree of predictability. In contrast, Metabolite 1, while energetically similar and finding a similar number of inhibitory poses, had many of these poses with only one or two members, suggesting significantly reduced predictability (Fig. 3A). Thus, Metabolite 1 is predicted to be an inferior proteasome inhibitor compared to (-)-ECG. This is verified by comparing their $\mathrm{IC}_{50}$ values against a purified proteasome $(3.43 \mu \mathrm{M}$ vs. $0.71 \mu \mathrm{M})$ (unpublished data).

Metabolite 2 adopts a pose that moves the ester carbon beyond the optimal distance for nucleophilic attack, $4.75 \AA$ away from the Thr 1 (Fig. 3B) and was found to possess an $\mathrm{IC}_{50}$ of $19.12 \mu \mathrm{M}$ unpublished data; Fig. 1A). Energetically, Metabolite 2 tended to bind with inferior energy than (-)-ECG (data not shown). Furthermore, the degree of predictability dropped further as Metabolite 2, paramethylated, found 41 different poses (Table I). Metabolite 2 found an inhibitory pose 40 times out of 100 , and found 15 different inhibitory poses (Table I). However, more than $80 \%$ of the poses were single member or low member solutions (data not shown), suggesting significantly reduced predictability in docking. This indicates that a single methylation in the para position on the D-ring can dramatically reduce the ability of (-)-ECG to inhibit the proteasome and that this may be due to the lack of a consistent binding pose by the metabolite and the inability of Thr 1 to attack the ester carbon.

Metabolite 3 is dimethylated on the D-ring. Dimethylation resulted in a dramatic change in docking pose (Fig. 3C). The ester carbon is moved distal from Thr 1 and the active site is filled by the stacking of the B- and C-rings, effectively blocking the ester carbon from Thr 1 and moving the ester carbon $6.58 \AA$ away from Thr 1 (Fig. 3C). Additionally, similar to Metabolite 2, Metabolite 3 did not settle into only a few poses. In fact, Metabolite 3 found 47 different binding poses, an inhibitory pose 48 times out of 100 and 23 different inhibitory poses (Table I), most of which were energetically inferior to (-)-ECG (data not shown). Additionally, the vast majority of poses were single solutions. This suggests a very low degree of predictability in binding to Thr 1 . Consistently the $\mathrm{IC}_{50}$ value of Metabolite 3 was determined to be 48.25 $\mu \mathrm{M}$ (unpublished data). These data suggest that dimethylation of (-)-ECG on the gallate group dramatically interferes with its ability to bind in the active site and to place its ester carbon near Thr 1 .

Monomethylated (-)-EGCG has decreased inhibitory pose predictability to the $\beta 5 \mathrm{Thr}$ 1. Unmethylated (-)-EGCG docks with 8 different poses and places the ester carbon in a good position to undergo nucleophilic attack 58 times out of 100 , and with only 4 different inhibitory poses (Table I). Monomethylation is capable of disrupting this docking but the disruption is moderate.

Metabolite 4 (Fig. 1B) is metamethylated on the D-ring and possesses a high probability of adopting a pose that places the ester carbon $3.00 \AA$ away from Thr 1 (Fig. 4A and Table I). 
A.

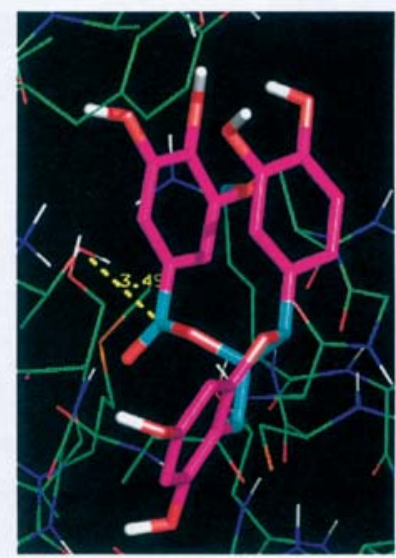

Metabolite 1

Distance: $3.49 \AA$
B.

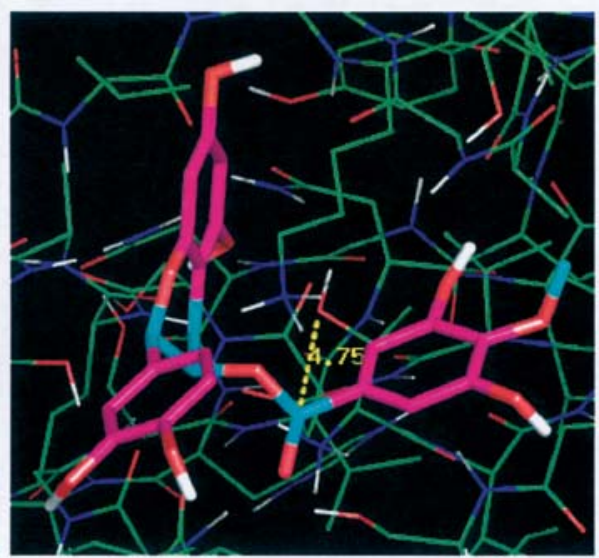

Metabolite 2

Distance: $4.75 \AA$
C.

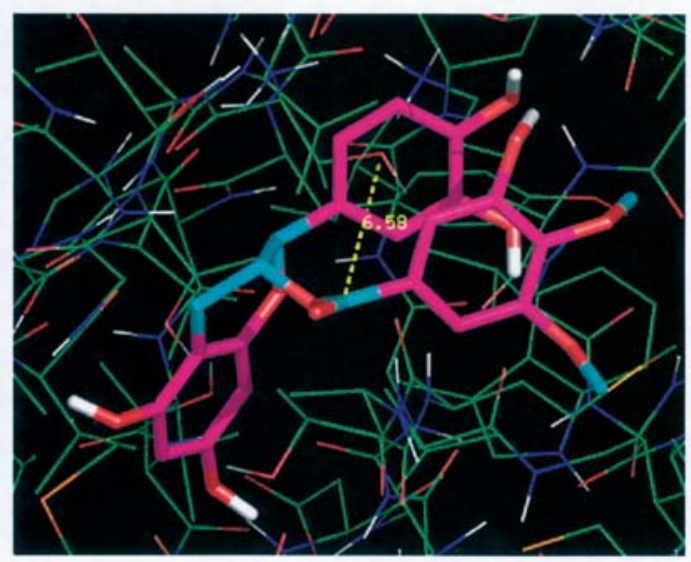

Metabolite 3

Distance: $6.58 \AA$

Figure 3. Changes in binding pose of (-)-ECG due to methylation on the D ring. (A) monomethylation on the meta position, Metabolite 1 has a modest effect on the positioning of (-)-ECG in the active site (distance from Thr 1,3.49 ̊; energy, -9.36) and a significant effect on predictability. (B) Paramethylation on the D ring, Metabolite 2, significantly pulls the ester carbon away from Thr 1 (distance from Thr 1, $4.75 \AA$; energy, - 7.20 ) to a distance unsuitable for nucleophilic attack. (C) Dimethylation, Metabolite 3, results in a pose in which the active site is filled by the stacking of the B and D rings (distance from Thr 1, 6.58 $⿱$; energy, -9.34), which moves the ester carbon distal to Thr 1 and the stacked rings occlude the carbon potentially blocking any reaction with Thr 1 .

A.

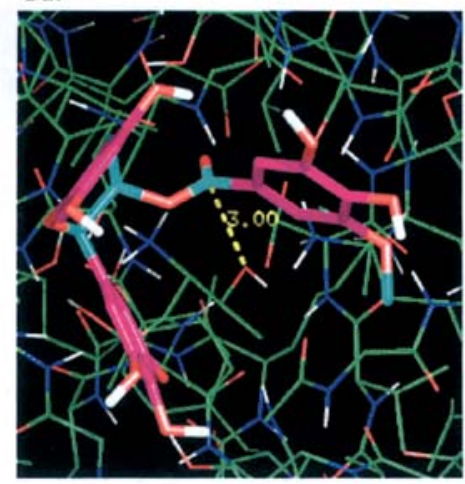

Metabolite 4

Distance: $3.00 \AA$
B.

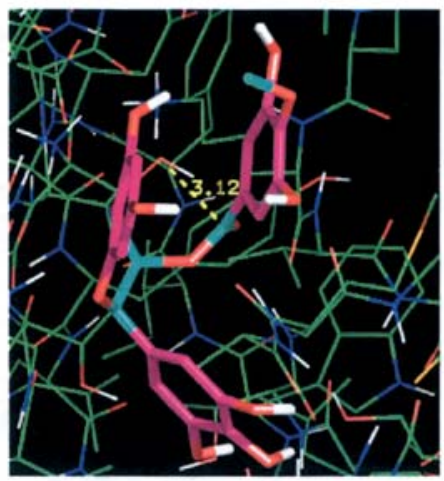

Metabolite 5

Distance: $3.12 \AA$

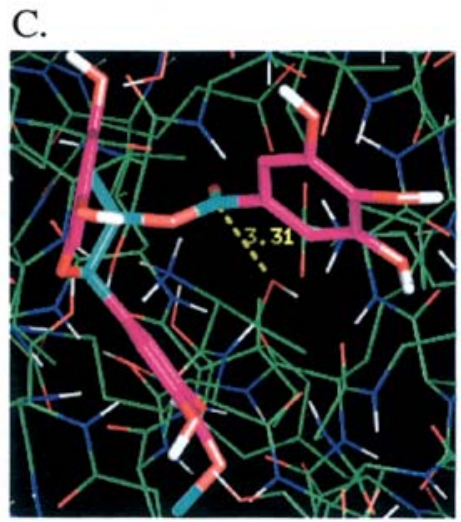

Metabolite 7

Distance: $3.31 \AA$
D.

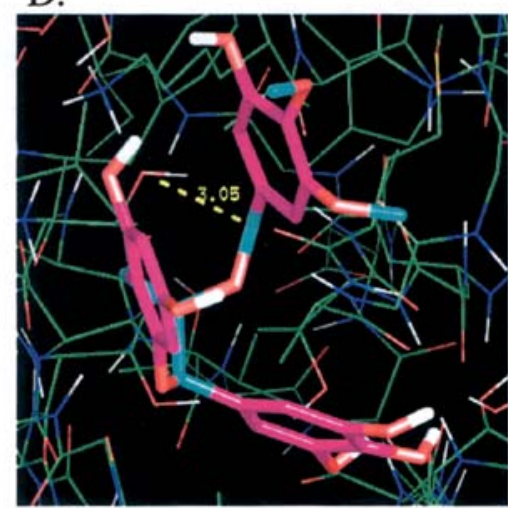

Metabolite 6

Distance: $3.05 \AA$

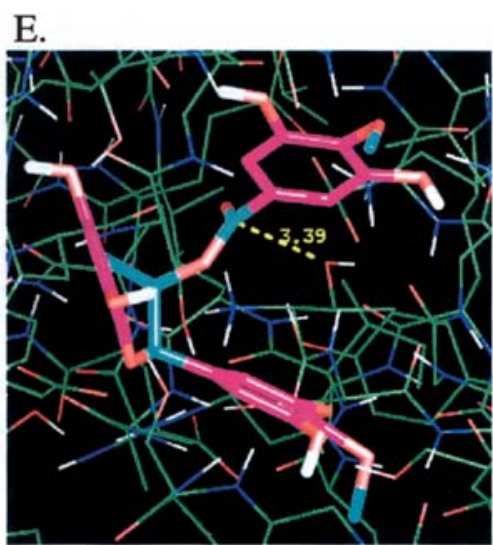

Metabolite 8

Distance: $3.39 \AA$

Figure 4. Changes in binding pose of (-)-ECGC due to mono or dimethylation. (A) Monomethylation on the meta position of the D ring, Metabolite 4, results

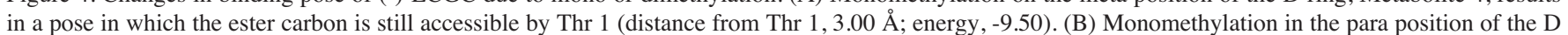
ring, Metabolite 5, affects the positioning of the metabolite similarly to metamethylation, though the ester carbon is further perturbed (distance from Thr 1, $3.12 \AA$ A ; energy, -9.13). (C) Metamethylation on the B ring, Metabolite 7, results in a position nearly identical to Metabolite 4, suggesting that this monomethylation has a modest effect on the ability of the compound to position the ester carbon near Thr 1 and a more pronounced effect on predictability when compared to (-)-EGCG (distance from Thr 1,3.31 ̊̊; energy, -8.17) (D). Dimethylation on the D ring, Metabolyte 6, moves the ester carbon similarly to monomethylation (distance from Thr 1,3.05 ̊̊; energy, -9.26). (E) Paramethylations on the B and D rings, Metabolite 8, does not disrupt the ability of the molecule to position the ester carbon near Thr 1 . Therefore, with dimethylation, the compounds can still adopt a pose that would facilitate inhibition of the proteasome (distance from Thr 1, $3.39 \AA$ A; energy, -9.32). However, methylation events significantly impact predictability of these poses (see Table I). 
A.

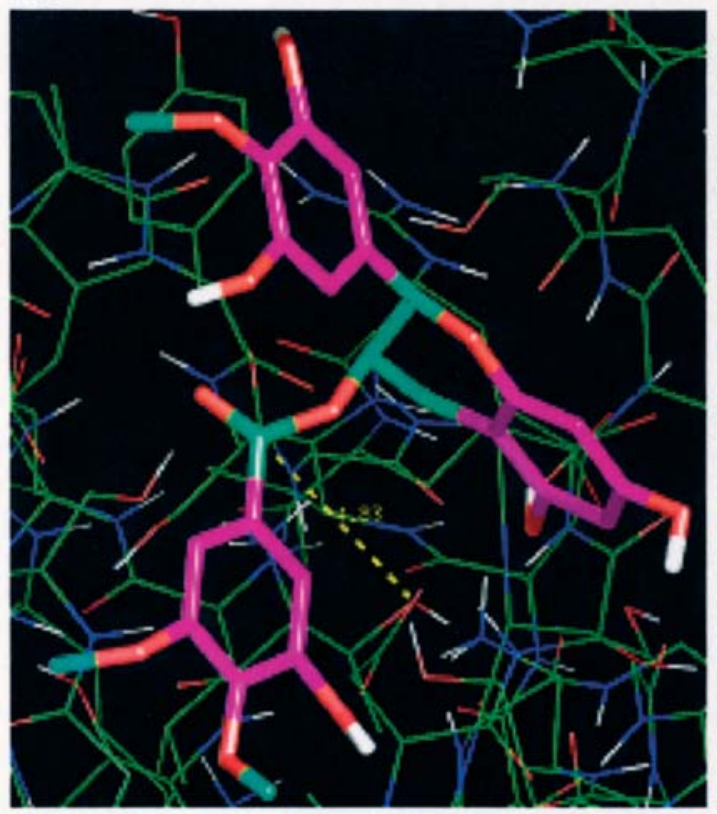

C.

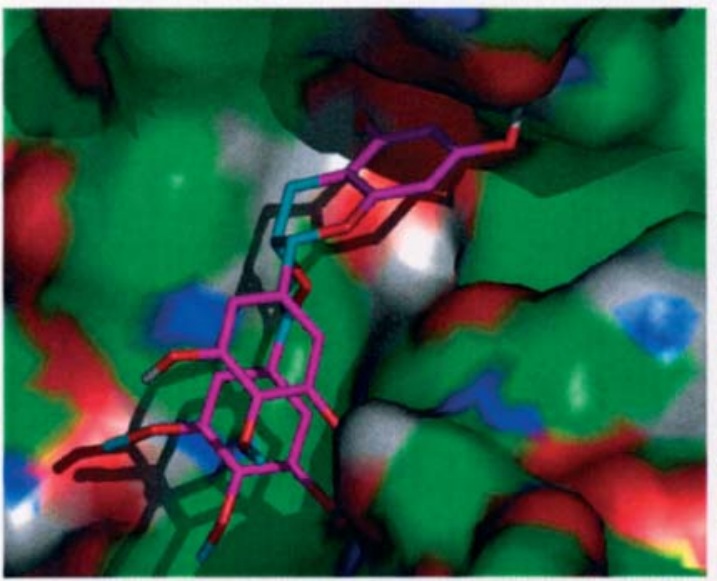

Metabolite 9 (A-D)
B.

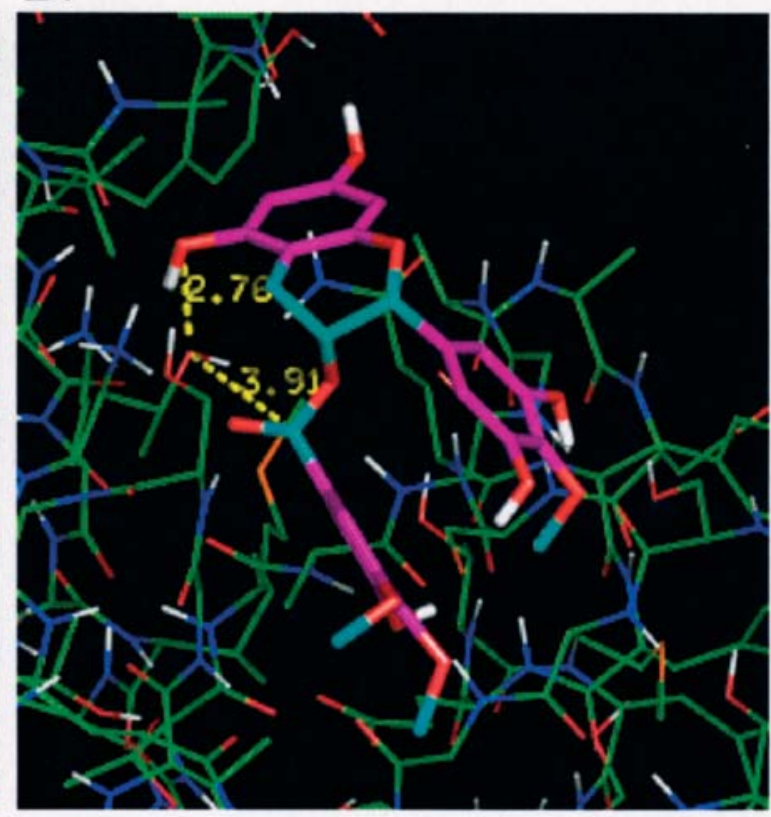

D.

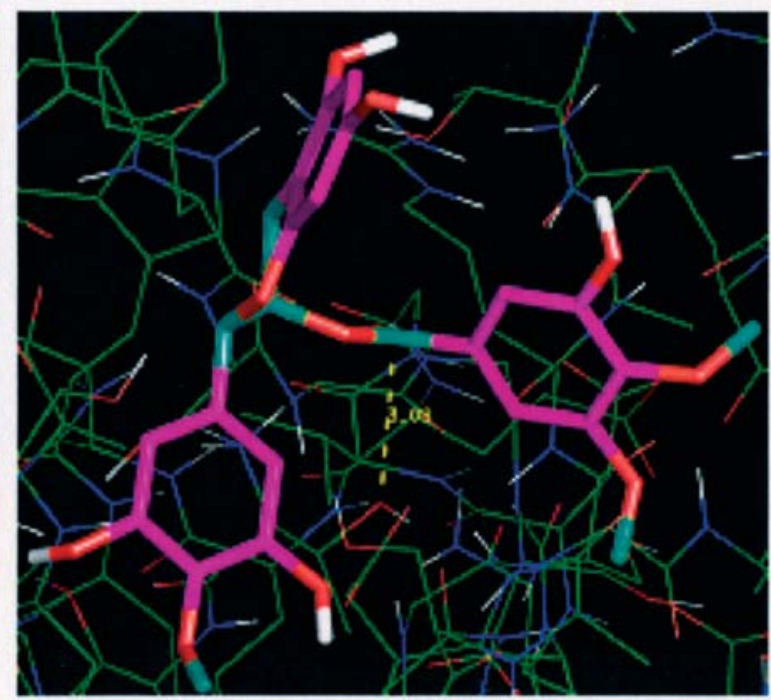

Figure 5. Trimethylation results in a pose that occludes the ester carbon, or promotes hydrogen bond formation between Thr 1 and (-)-EGCG. (A-C) Example poses adopted by Metabolite 9 from multiple docking runs, suggesting the majority of poses places the A/C ring system proximal to Thr 1 allowing for hydrogen bond formation. (C) Image depicting the surface of the active site showing the A/C rings filling the pocket. (D) A minority pose that would allow Thr 1 to interact with the ester carbon.

This metabolite found 32 different poses, an inhibitory pose 56 times out of 100 and 16 different inhibitory poses (Table I and Fig. 4A). Comparatively, Metabolite 4 finds more poses and adopts the inhibitory poses approximately as often as (-)-EGCG (Table I). The $\mathrm{IC}_{50}$ of Metabolite 4 was found to be $5.63 \mu \mathrm{M}$ (unpublished data; Fig. 1B), significantly less potent than (-)EGCG $\left(\mathrm{IC}_{50}\right.$ of $\left.0.21 \mu \mathrm{M}\right)$ and consistently, the dock was significantly less predictable than (-)-EGCG.

Metabolite 5, paramethylated on the D-ring of (-)-EGCG, does not significantly move the ester carbon away from Thr 1, measured as $3.12 \AA$ (Fig. 4B). Metabolite 5 found 28 different poses, an inhibitory pose 46 times out of 100 and 8 different inhibitory poses (Table I), suggesting a binding that is similarly predictable, if perhaps slightly inferior, to Metabolite 4 . The similarly predictable binding and proximal position of the ester carbon to Thr 1 correlates to Metabolite 5's similar $\mathrm{IC}_{50}, 6.91 \mu \mathrm{M}$ compared to the $5.63 \mu \mathrm{M}$ of Metabolite 4 (unpublished data; Fig. 1B).

Metabolite 7 is paramethylated on the B ring (Fig. 1). This monomethylation has little effect on the positioning of the ester carbon in regards to Thr $1,3.31 \AA$ (Fig. 4C). Metabolite 7 found 24 different poses and 69 out of 100 poses were inhibitory, which is slightly better than Metabolites 4 and 5 (Table I). However, it found 8 different inhibitory poses (Table I). This suggests a more stable dock than Metabolite 4, but is still worse than (-)-EGCG. Metabolite 7 
was found to have a superior $\mathrm{IC}_{50}$ value $(2.45 \mu \mathrm{M}$, Fig. 1B) than Metabolite 4 or 5 , suggesting that methylation on the $\mathrm{B}$ ring has a less significant impact than methylation on the $\mathrm{D}$ ring.

The positioning of the ester carbon and the predictability data support the biological $\mathrm{IC}_{50}$ values. Metabolites 4 and 5 are very similar, though Metabolite 4 is slightly more potent, and Metabolite 7 is superior to both. This suggests that a single methylation may dramatically worsen the $\mathrm{IC}_{50}$ of (-)-EGCG but it does not seem to be enough to completely disrupt the ability of the molecule to find a favorable inhibitory pose.

Dimethylation of (-)-EGCG has more effect on binding than monomethylation. Metabolite 6 is dimethylated on the D ring and Metabolite 8 is monomethylated on both the $\mathrm{D}$ ring and the $\mathrm{B}$ ring (both in the para position, Fig. 1B). In each case, the principle pose places the ester carbon within the $4 \AA$ distance necessary for nucleophilic attack (Fig. 4D and E). Metabolite 6 adopts 35 poses with 64 out of 100 as possibly inhibitory and 15 different inhibitory poses (Table I). Furthermore, several inhibitory poses may be blocked by potential hydrogen bonds formed by the oxygen of Thr 1 (data not shown). These data suggest that Metabolite 6 would be predicted to be slightly less potent than Metabolites 4 and 5, which correlates with their in vitro $\mathrm{IC}_{50}$ values $(9.81 \mu \mathrm{M}$ vs 5.63 and $6.91 \mu \mathrm{M}$ ) (unpublished data; Fig. 1B).

Metabolite 8 adopts 34 poses with 55 out of 100 as possibly inhibitory and 10 different inhibitory poses (Table I). Similar to Metabolite 6, several inhibitory poses may be blocked by hydrogen bond formation. These data show that the second methylation further disrupts the binding but only slightly more than monomethylation. This suggests that Metabolite 8 in slightly less potent than Metabolites 4 and 5 and comparable to Metabolite 6, which correlates with their in vitro $\mathrm{IC}_{50}$ values $(8.23 \mu \mathrm{M}$ vs. 5.63, 6.91 and $9.81 \mu \mathrm{M}$, respectively) (unpublished data; Fig. 1B).

Trimethylation almost completely disrupts the ability of (-)EGCG to bind into the active site. Metabolite 9 is a trimethylated (-)-EGCG analog with 2 methyl groups on the D ring and 1 methyl group on the B ring (Fig. 1B). The most common pose adopted, moved the ester carbon more than $4 \AA$ and/ or the active site was filled with the A- and C-ring system (Fig. 5A-C). One variant of this pose placed the ester carbon $3.91 \AA$ from Thr 1 (Fig. 5B). However, similar to Metabolites 6 and 8 , Metabolite 9 tended to dock so that a hydroxyl group from the A/C-ring system is proximal to Thr 1 (2.76 $\AA$, Fig. 5B). Therefore, the potential for hydrogen bond formation between the hydroxyl of Thr 1 and the hydroxyl of (-)-EGCG may be a greater driving force than nucleophilic attack on the ester carbon for the poses this molecule adopts. Another pose suggested a possible position that could lead to nucleophilic attack but appears to be in the minority of poses adopted by Metabolite 9 (Fig. 5D). The probability of a predictable pose is highly compromised. Metabolite 9 adopts 40 different poses, and finds an inhibitory pose 64 out of 100 times (subject to the possible hydrogen bond and ring occlusions mentioned above), but the vast majority of solutions are single member or low member poses (data not shown), suggesting a significant lack of predictability. Due to the lack of pose consistency, additional docking runs were performed with Metabolite 9, which further validated the above observation. These data, extreme lack of predictable binding, blocking the ester carbon with a potential hydrogen bond from another hydroxyl, and interference from ring stacking suggest that Metabolite 9 would be a poor proteasome inhibitor. This is confirmed by its $\mathrm{IC}_{50}$ of $43.03 \mu \mathrm{M}$ (unpublished data; Fig. 1B).

\section{Discussion}

Results from a recent case-control study suggest that methylated polyphenols are less cancer-protective (22). However, the molecular basis for this observation is unknown. We hypothesize that methylation could decrease the proteasome-inhibitory abilities of the green tea polyphenols. Indeed, methylation of (-)-ECG and (-)-EGCG results in a dramatic increase in $\mathrm{IC}_{50}$ values by up to 205 -fold (Fig. 1; unpubliched data). However, methyation events do not seem to be related to a loss of nucleophilic susceptibility at the ester carbon (Fig. 2). The data for the docking of methylated (-)-ECG and (-)-EGCG analogs suggest that steric blocking of the active site may be insufficient to inhibit proteasome activity and that covalent modification may be the principle means by which these compounds inhibit proteasome activity (10). Therefore, polyphenol analogs that do not have predictable docks and/or dock in poses that block the ester carbon or move the ester carbon away from Thr 1 may have dramatically reduced efficacy against the proteasome.

The placement of the methylation in the case of (-)-ECG is significant with respect to the position of the ester carbon in that the ester carbon was moved away from or blocked entirely from Thr 1 (Fig. 3). This strongly suggests that methylation at the para site on the $\mathrm{D}$ ring, or multiple methylation events can nearly negate the ability of (-)-ECG-based compounds to inhibit the proteasome.

Previously, we showed a pose that allowed (-)-EGCG to predictably fit in the active site of the proteasome 35 subunit in such a manner as to promote nucleophilic attack on the ester carbon (10). It became apparent, after docking the methylated analogs, that none of these compounds adopted a very similar pose, nor did they consistently cluster with respect to the docking poses they adopted when compared to unmethylated (-)-EGCG (Figs. 4 and 5) (10). In the case of monomethylation, the position or ring that was methylated seemed to have a moderate influence on the $\mathrm{IC}_{50}$ value of each compound (Fig. 4). This correlates to the general predictability of binding by these compounds and that the poses adopted by these compounds place the ester carbon proximal to Thr 1 .

However, additional methylation, especially trimethylation, seems to have a dramatic impact on the ability of these compounds to bind with the ester carbon available for attack and predictably. Dimethylation worsens the binding that was already impaired in the case of monomethylation (Fig. 4). Trimethylation almost completely disrupts the ability of the compound to bind predictability and the ester carbon could be blocked from Thr 1 by the hydroxyl on the A/C-ring system (Fig. 5). This may account for the poor proteasomeinhibitory activity evidenced by Metabolite 9 (unpublished data). 
Biotransformation reactions, including methylation (23), lead to reduced biological activities of (-)-EGCG in vivo $(13,14)$. However, the molecular basis for how methylation reduces the biological activities of (-)-EGCG remains unclear. In the current study, we found that single methylation events on the B-ring or at the meta position on the D ring may have moderate reducing effects on the potency of the tea polyphenols, which was further increased by di- or trimethylations.

Using in silico docking methods, tea polyphenol analogs can be tested for their ability to place the critical ester carbon proximal to Thr 1 and in an unblocked conformation. Further analysis of docking predictability could also prove beneficial. This iterative process may help find theoretical analogs that can serve as leads for potential anticancer drugs and examine why some compounds may fail. In the absence of crystal structures and with validation by biological experiments, these methods may help explain the changes in the activity of methylated tea polyphenols in regards to the proteasome and other potential targets. This may assist in understanding the full effects of tea polyphenols and their methylated metabolites in cancer.

\section{Acknowledgements}

This work was supported by research grants from the National Cancer Institute-National Institutes of Health and Barbara Ann Karmanos Cancer Institute Prevention Program (to Q.P.D.), National Cancer Institute-Ruth L. Kirschstein National Research Service Award (to K.R.L.P.), and the Areas of Excellence Scheme established under the University Grants committee of the Hong Kong Special Administrative Region, China (to T.H.C., Project No. AoE/P-10/01).

\section{References}

1. Ahmad N and Mukhtar H: Green tea polyphenols and cancer: biologic mechanisms and practical implications. Nutr Rev 57: 78-83, 1999.

2. Fujiki H: Two stages of cancer prevention with green tea. J Cancer Res Clin Oncol 125: 589-597, 1999.

3. Yang CS: Tea and health. Nutrition 15: 946-949, 1999.

4. Lambert JD and Yang CS: Mechanisms of cancer prevention by tea constituents. J Nutr 133: 3262S-3267S, 2003

5. Lambert JD and Yang CS: Cancer chemopreventive activity and bioavailability of tea and tea polyphenols. Mutat Res 523-524: 201-208, 2003.
6. Kuhn DJ, Burns AC, Kazi A and Dou QP: Direct inhibition of the ubiquitin-proteasome pathway by ester bond-containing green tea polyphenols is associated with increased expression of sterol regulatory element-binding protein 2 and LDL receptor. Biochim Biophys Acta 1682: 1-10, 2004.

7. Chen D, Daniel KG, Kuhn DJ, Kazi A, Bhuiyan M, Li L, Wang Z, Wan SB, Lam WH, Chan TH and Dou QP: Green tea and tea polyphenols in cancer prevention. Front Biosci 9: 2618-2631, 2004.

8. Lam WH, Kazi A, Kuhn DJ, Chow LM, Chan AS, Dou QP and Chan TH: A potential prodrug for a green tea polyphenol proteasome inhibitor: evaluation of the peracetate ester of (-)epigallocatechin gallate [(-)-EGCG]. Bioorg Med Chem 12: 5587-5593, 2004

9. Nam S, Smith DM and Dou QP: Ester bond-containing tea polyphenols potently inhibit proteasome activity in vitro and in vivo. J Biol Chem 276: 13322-13330, 2001.

10. Smith DM, Daniel KG, Wang Z, Guida WC, Chan TH and Dou QP: Docking studies and model development of tea polyphenol proteasome inhibitors: applications to rational drug design. Proteins 54: 58-70, 2004.

11. Crespy V and Williamson G: A review of the health effects of green tea catechins in in vivo animal models. J Nutr 134: 3431S-3440S, 2004.

12. Greenwald P: Clinical trials in cancer prevention: current results and perspectives for the future. J Nutr 134: 3507S-3512S, 2004.

13. Zhu QY Zhang A, Tsang D, Huang Y and Chen ZY: Stability of green tea catechins. J Agric Food Chem 45: 4624-4628, 1997.

14. Okushio K, Suzuki M, Matsumoto N, Nanjo F and Hara Y: Methylation of tea catechins by rat liver homogenates. Biosci Biotechnol Biochem 63: 430-432, 1999.

15. Berman HM, Westbrook J, Feng Z, Gilliland G, Bhat TN, Weissig H, Shindyalov IN and Bourne PE: The Protein Data Bank. Nucleic Acids Res 28: 235-242, 2000.

16. Groll M, Ditzel L, Lowe J, Stock D, Bochtler M, Bartunik HD and Huber R: Structure of $20 \mathrm{~S}$ proteasome from yeast at $2.4 \mathrm{~A}$ resolution. Nature 386: 463-471, 1997.

17. Kisselev AF and Goldberg AL: Proteasome inhibitors: from research tools to drug candidates. Chem Biol 8: 739-758, 2001.

18. Dym O, Xenarios I, Ke H and Colicelli J: Molecular docking of competitive phosphodiesterase inhibitors. Mol Pharmacol 61: 20-25, 2002.

19. Rao MS and Olson AJ: Modelling of factor Xa-inhibitor complexes: a computational flexible docking approach. Proteins 34: 173-183, 1999

20. Morris GM, Goodsell DS, Halliday RS, Huey R, Hart WE, Belew RK and Olson AJ: Automated docking using a Lamarckian genetic algorithm and an empirical binding free energy function. J Comput Chem 19: 1639-1662, 1998.

21. DeLano WL: The PyMOL Molecular Graphics System, DeLano Scientific, San Carlos, CA, 2002.

22. Wu AH, Tseng CC, Van Den Berg D and Yu MC: Tea intake, COMT genotype, and breast cancer in Asian-American women. Cancer Res 63: 7526-7529, 2003.

23. Zhu BT, Patel UK, Cai MX and Conney AH: O-Methylation of tea polyphenols catalyzed by human placental cytosolic catechol-O-methyltransferase. Drug Metab Dispos 28: 1024-1030, 2000 . 